Julia Schreiner

Neue (Auf)Schreibsysteme. Verändern
Weblogs die Konventionen des
geschichtswissenschaftlichen Schreibens?

Blogs von WissenschaftlerInnen, die nicht rein privater Natur sind, sind Teil von Wissenschaftskommunikation und Teil des (Auf)Schreibsystems Wissenschaft. Verändert sich durch die Schreibform Bloggen das Schreiben von Geschichte an sich? Oder zurückhaltender formuliert: Welche Chance für Veränderungen sind dem Medium Blog eigen? Entlang der Querschnitte Subjektivierung von Schreiben, Kollektivierung von Schreiben, Schreiben in zeitlicher Dimension werde ich im Folgenden dieser Frage nachgehen - und dabei noch einen Seitenblick werfen auf gewisse Tendenz zu Monopolisierungen im Schreib-System Blog.

\title{
Das Ich
}

„Die Beiträge werden sich auf neu erschienene oder von mir (wieder-)entdeckte Bücher oder einzelne Aufsätze und Zeitungsartikel beziehen, auf Berichte in TV und Radio." So schreibt Jan Hecker-Stampehl über seinen Blog nordic history (nordichistoryblog.hypotheses.org/70). Das Zitat ist exemplarisch für viele andere Beschreibungen der eigenen Blogs. Es geht darum, was der Autor, die Autorin, schreiben möchte. Es geht darum, explizit die eigene Meinung zu formulieren. Über die Schreib- und Publikationsform Blog wird das Ich als Ausdruck von Subjektivität gestärkt, das verschleiernde man rückt ins Abseits.

Im Schreibsystem Weblog ist das Ich nicht nur legitim, sondern sogar unabdingbar. „Ich“ zu sagen, gehört zum Bloggen dazu, weil es sich hier um Schreiben im ursprünglichen Sinne des Weblogs, des Online-Tagebuches handelt. Wirkt dieses Ich-Sagen zurück auf das wissenschaftliche Schreiben auch außerhalb Blogosphäre? Wird über den (Um-)Weg der Weblogs allgemein deutlicher, dass auch wissenschaftliche Texte von persönlichen Perspektiven, Fragestellungen und Sprachen geprägt sind?

Offensichtlich bedeutet Bloggen nicht die Beerdigung der Autorschaft. Folgen wir dem Rat von Marc Scheloske (Vortrag auf der Kick-Off-Tagung von de.hypothese; siehe www.scheloske.net/ und www.wissenswerkstatt.net/2012/ 03/13/wege-aus-der-nische-was-man-von-erfolgreichen-wissenschaftsblogslernen-kann/), einem frühen Pionier des wissenschaftlichen Bloggens, dann 
muss ein Blog sogar immer personenbezogen sein und sich durch eine unbedingt charismatische Autorpersönlichkeit auszeichnen - zumindest wenn dieser Blog ein erfolgreicher Blog sein soll.

Kann Charisma eine Grundanforderung an wissenschaftliches Schreiben sein? Ist das Ich-Sagen also nicht nur in methodisch-reflexiver Hinsicht ein Unterschied zu traditionellen Publikationsformen? Sondern geht es unter so einem Blickwinkel rasch mehr um Selbstdarstellung als um Inhalte (siehe Beitrag von Anton Tantner)? Selbstdarstellung meint dann die Darstellung des eigenen Selbst, des Ich des Schreibenden, meint aber auch Darstellung im Sinne von Präsentation. Traditionelle Publikationsformen grenzen z. B. die Möglichkeiten, die Optik des eigenen Beitrages zu beeinflussen, weitgehend ein: Schrift und Satzbild einer Zeitschrift sind vorgegeben, die Redaktion bestimmt, wann und wie ein Aufsatz publiziert wird. Im Weblog hingegen sind AutorIn und Publikationsort eins.

Der oben zitierte Weblog "nordic history“ hat sich unterdessen zum Gemeinschafts-Blog gewandelt - von Scheloske überspitzt formuliert eigentlich ein „No-Go", in der Wissenschaftskommunikation jedoch ein probates Mittel, um zu einem Thema verschiedene spannende Impulse zu versammeln.

\section{Das Wir}

Liegt in den Gemeinschaftsblogs schon das Ende des Ich - der Anfang des Wir? Ja und Nein. Ja, denn mit jeglichem Gemeinschafts-Blog ist die eineindeutige Übereinstimmung der Urheberschaft des/der Bloggenden mit der URL des Blogs gebrochen. Mehr aber auch nicht. Das ist somit weder das Ende des Ichs, noch der Anfang des Wir.

Denn auch in Gemeinschafts-Blogs können mehrere Ichs autonom schreiben. Frühes, im deutschsprachigen Raum bekanntestes Beispiel ist das Blog von Peter Haber und Jan Hodel auf hist.net (weblog.hist.net/). Hier schreiben (bzw. seit April 2013 nun leider: schrieben) beide Autoren mit ihrer je eigenen „Handschrift" Beiträge, zumindest in den letzten zwölf Monaten gab es keinen Gemeinschaftstext mehr und zum Autoren-Kollektiv wird hist.net vor allem über gegenseitige Kommentierungen.

Kommentare sind eine Spur in Richtung kollektiven Schreibens, in Richtung des Wir. Das ist ja eines der Versprechen der Blogosphäre, dass durch vielfältiges gegenseitiges Kommentieren Formen gemeinschaftlichen Schreibens entstehen, Formen, die letztlich zu besseren Texten führen. Und vom Austausch mancher Sticheleien abgesehen, wird in den meisten Fällen kollegiale Kritik kollegial und freundschaftlich beantwortet. Dass zur Blogosphäre auch die 
Ausnahmen zählen, ist leider ebenso unvermeidbar, wie der Umstand, dass diese Ausnahmen ggf. besonderes Gewicht sowohl innerhalb der Sphäre als auch in deren Außenwirkung erlangen. Es gibt in der Community auch noch keinen basalen Konsens, was die Mindeststandards von wissenschaftlicher Kommunikation sind. Eine „Netiquette“ wurde bisher nicht aufgestellt, weder von der European Association for Digital Humanities, noch von der europäischen Blog-Plattform hypotheses.org. Ebenso wenig gibt es eine Verabredung, wie mit diffamierenden Texten umzugehen ist. Seltsamer Weise scheint im Gegenteil häufig noch die Annahme vorzuherrschen, dass im Zweifel inhaltlich wertvolle Beiträge verbale Entgleisungen überstrahlen (adresscomptoir. twoday.net/stories/156272066/). Dies führt zur Grundfrage nach Seriosität und Ernsthaftigkeit (was nicht dasselbe ist) von Weblogs. Mit dieser Frage steht und fällt aber auch der Einfluss, den die Textform Blog auf das gesamte wissenschaftliche Schreiben wird nehmen können.

Von diesem Umweg zurück zum Schreib-Netz-Werk der Blogosphäre. Das System des Kommentierens ist nicht nur eines des Schreibens, sondern eines des Lesens und Schreibens. Dies mag es als gegenseitiges Zitieren, Rezensieren und in Sammelbände Einschreiben auch schon in analogen oder frühdigitalen Zeiten gegeben haben. Alle originär digitalen Schreibformen machen die Gedanken-Netzwerke indes noch klarer sichtbar. Gerade die Multiplikation von Inhalten über den Mikro-Blog Twitter verdeutlicht dies. Entscheidend in diesem Netzwerk sind noch nicht einmal die geposteten Kommentare - hier wird bisweilen eher noch ein Mangel beklagt (Landes: rkb.hypotheses.org/194 oder auch der Beitrag von Mareike König in diesem Band), sondern die Verlinkungen, Pingbacks und dergleichen, die das eigentliche Netz der Kommunikation stricken. Interessant ist in diesem Zusammenhang für mich auch der Hinweis von Stefan Hessbrüggen (im Kommentar [sic!] zum gerade genannten Beitrag), dass die Diskussion von Blogs in Sozialen Netzwerken eine andere Art und Weise der Kommentierung sei, die die Hoheit über die Inhalte jedoch anders verteile. Ist der Kommentar einmal abgesetzt, kann er in der Regel nicht mehr gelöscht werden - eine kommentierte Verlinkung auf einen anderen Beitrag im eigenen Blog hingegen natürlich schon.

Anders gefragt: Vielleicht sind Kommentare per se überschätzt? „The comment feature of blogs is vastly overrated anyway. My back-of-the-envelope calculation is that $1 \%$ of blog comments are useful to other readers", postulierte Dan Cohen 2005 in seiner Anleitung, ein eigenes Weblog anzulegen. Seit Sommer 2007 - als er mit seinem Blog auf WordPress umstieg - lässt allerdings auch Dan Cohen Kommentare zu. Die Möglichkeit einer Reaktion, die direkt für alle, die den Blog lesen, sichtbar ist, hat vielleicht überzeugt.

Noch stärker und noch transparenter als beim Bloggen wird die kollektive Arbeit an einem Text in der Spielart des Open Peer Review, wie sie dem 
Projekt „historyblogosphere“ zugrunde liegt. Besonders im Feld der naturwissenschaftlichen Fachjournale ist dies bereits ein erprobtes Mittel - hier nur ein Beispiel: „Atmospheric Chemistry and Physics" www.atmospheric-chemistryand-physics.net/. Der Wegweiser in Richtung eines kollektiven und transparenten Schreibprozesses ist aufgestellt, auch die Schreib-Projekte „Writing History in the Digital Age“ sowie ganz neu „Open Review“(http://openreview.net/) folgen dieser Idee, die offensichtlich von der Interaktion von Schreibenden und Lesenden, wie sie die Blogosphäre ermöglicht hat, profitiert.

Das Wir im Schreib-System Blog meint wie im Open Peer Review das Transparentmachen von Einflüssen, die deutlich sichtbarer werden als bei Zitationen. Es meint nicht so sehr, das unter einer URL zusammengefasste AutorInnenkollektiv, das letztlich doch aus einzelnen Schreibenden besteht, die sich gemeinsam einem Thema verschreiben. In diese Kategorie fallen auch die vielfältigen Projekt-bezogenen (z. B. ehenvorgericht.wordpress.com/) oder institutionellen (z. B. www.maxweberstiftung.de/) Blogs.

\section{Schreiben als Prozess - oder Abschied von der Endgültigkeit}

Das Weblog als Schreibsystem ist - eigentlich - ein Schreibprozess. Es besteht zumindest die Option, dass nicht nur der fertige Text, nicht das Produkt veröffentlicht wird, sondern dieser Prozess. Das obliegt aber vor allem der persönlichen Arbeitsweise der Bloggenden. Damit ist auch ein öffentliches Scheitern des Schreibprojekts als Möglichkeit inbegriffen.

Aber dieses Postulat gilt eben nur eigentlich: Bei allen institutionell betriebenen Blogs ist diese Eigenart per se ausgeblendet. Und auch bei den Blogs, die sich weitgehend auf Einzel-Posts konzentrieren, greift die vierte Dimension nur in reduzierter Variante, nämlich in der Hinsicht, dass etwa Gedanken aus dem Netz(werk) aufgegriffen werden, und dann - oft in Form von eigenen Kommentaren zum eigenen Text - weitergeführt werden.

Denn auch die Einträge in Weblogs sind nicht Schreiben direkt aus den Gedanken; sondern in den meisten Fällen Texte, die erst in einem privaten, abgeschlossenen (Daten-)Raum aufgesetzt, überdacht, umgearbeitet werden, bevor sie veröffentlicht werden.

Dies ist wahrscheinlich ein Grundunterschied zum Kleinst-Text-Format Twitter. Die Texte der Weblogs sind längst der Dauerhaftigkeit verpflichtet und sie sind zunehmend Teil der eigenen digitalen Visitenkarte (siehe Beitrag von Anton Tantner). Sehen wir Twitter als eine Form von Mikro-Blogging, dann zeichnet diese Schreibart viel stärker das Akute, Gedrängte, auch Kryptische 
aus - vor allem beim Live-Twittern zu Tagungen zu beobachten (weblog.hist. net/archives/6100). Und auch die Geschwindigkeit, mit der auf Tweeds reagiert, geantwortet, re-tweeted wird, ist eine ganz andere. Bei Tagungen hat diese Schreibart bisweilen etwas von Kritzeleien im Klassenzimmer - allerdings mit dem Unterschied einer zumindest potentiellen Öffentlichkeit. Generell wird der Mikro-Blog auch von HistorikerInnen immer stärker eingesetzt, er ist die Möglichkeit, um Informationen rasch mit dem eigenen Netzwerk zu teilen.

Geschichtswissenschaftliche wie auch andere Blogs werden zwar bewahrt ggf. sogar langzeit-archiviert (in Frankreich nun sogar über die Bibliothèque nationale, wie M. König in ihrem Beitrag in diesem Band schreibt), geschrieben werden sie zumeist dennoch als Momentaufnahmen.

Als Schlussfolgerung ist diese Eigenart also nicht weiter zu kritisieren, sondern als solche ernst zu nehmen und zu schätzen. Denn im Unterschied zu Print-Publikationen stehen beim Bloggen keine Schranken vor den Toren der Öffentlichkeit, und auch der zeitliche Ablauf vom Gedanken zum veröffentlichten Text ist allein der Arbeitsweise des Autors, der Autorin geschuldet.

Das heißt nicht, dass Blogs nicht auch als fortlaufende, fortgesetzte Texte geschrieben werden können. Gerade die nicht-wissenschaftlichen Weblogs, sehr früh etwa das von Rainald Götz („Abfall für alle“, unterdessen nur noch als gedrucktes Buch [sic!] einzusehen), haben diesen Weg beschrieben. Spannend sind hier die vielen derzeit neu gegründeten Blogs zu Dissertations- und Forschungsprojekten, die offen die Fragen der eigenen Forschung zu diskutieren suchen. Noch spannender ist ein Blick hinaus aus der deutschsprachigen Blogosphäre, etwa zur florierenden angelsächsischen. Hier finden sich am ehesten Blogs, die der Idee Mills Kellys folgen oder eher vorgreifen (switzerland2011. thatcampdev.info/session-reports/thatcamp-sessions/hows-changing-thewriting-of-history/), ganze Geschichten fortzuschreiben: Von den ersten Recherchen im Archiv bis zum fertigen - oder verworfenen - eigenen Text. Dies demonstriert im Sinne einer thematischen Engführung mit ausführlicher methodischer Reflexion Newton Key auf: earlymodernengland.blogspot.co.uk/. Keys Blog zeichnet sich aus durch ausgefeilte Beiträge, die Bilder wirklich kommentieren, zur Diskussion stellen - und nicht nur flüchtig aneinander reihen mit dem Hauptziel möglichst viele Blog-Einträge zu kreieren (die Blogosphäre zur spannenden Diskussion um die „Frühe Neuzeit“ erklärt uns Newton Key übrigens in seinem Beitrag hier in diesem Band).

Hilfreich beim Auffinden neuer Weblogs ist die Rubrik „Guck mal, wer da bloggt", die regelmäßig die Neuzugänge auf de.hypotheses vorstellt (zuletzt: redaktionsblog.hypotheses.org/622). Besonders gespannt war ich auf die in die Kategorie „eine Dissertation begleitend“ einsortierten. Ich hatte mir streng den Forschungs- und Schreibprozess begleitende, reflektierende, ja fortschreibende Weblogs, erhofft. Auf den ersten (sic!) Blick aber finde ich weitgehende Ge- 
mischtwarenläden aus Vortragsankündigungen und dergleichen. Das spricht nicht gegen diese Blogs; nur meine Erwartung wurde enttäuscht. Und dennoch, wenn ich die Ankündigungseinträge ausblende - oder im Zweifel auch den guten Strukturen/Gliederungen folge -, dann fügt sich bisweilen doch etwas zusammen, was als Weblog einer Dissertation sehr gut funktionieren kann; auch als Aufschreibsystem im Sinne einer freieren Publikationsform, die die eigenen Gedanken zum eigenen Forschungsfeld schon früh in Worte und - vor allem - ganze Sätze fasst. In Sätze, die andere lesen (können) und somit öffentlich sind. Zu nennen sind etwa: Mark Mudrak: catholiccultures.hypotheses. org/dissertationsprojekt. Und besonders markant ist Robert Zimmermann im Eintrag über sein Blog: „im Sinne eines ,Versuchslabors' soll dieser Blog Fragestellungen aufgreifen, die mir in meinem Forschungsalltag begegnen. Dabei sollen Thesen vorgestellt, Gedanken zu relevanter Literatur veröffentlicht und Veranstaltungshinweise gegeben werden." (umstrittenesgedaechtnis. hypotheses.org/uber)

Oder auch Andreas Lerch im Eintrag über seinen Blog Astrologie in der Frühen Neuzeit: „Neben der rein historischen Analyse möchte ich meine wissenschaftstheoretischen Gedanken hier notieren und zur Diskussion stellen." (astrologiefnz.hypotheses.org/35)

Es zieht also die Kategorie des Vorläufigen ein ins wissenschaftliche Schreiben - und Publizieren. Es verändert sich die Zeitlichkeit der Texte.

\section{Die Macht der Technik}

So wie die derzeit noch ungebrochene Dominanz von Powerpoint Präsentationen vorbestimmt, so werden auch die Strukturen von Blogs und die Systeme der zum Blog zugehörigen Kommunikation durch ihren technischen Rahmen geprägt. Natürlich kann jeder und jede mit dem entsprechenden Aufwand individuelle Konfigurationen bauen. Denn die derzeit dominanten Programme und Plugins, vor allem die zur WordPress-Familie gehörenden, sind allesamt Open-Source-Produkte. Das derzeitige Versprechen tönt jedoch gerade: Jeder und jede kann mit-machen, mit-schreiben - und dies ohne großen Aufwand, und ohne erhebliche Technik-Kenntnisse.

Es geht freilich nicht darum, eine (derzeit) dominante Software zu verteufeln. Vielmehr sind die Auswirkungen der technologischen Rahmensetzungen auf das Schreiben von Geschichte zu analysieren. Insofern ist nicht nur eine Methode, sondern auch eine Theorie der digitalen Geschichtsschreibung gefordert - auch wenn das vielleicht weniger "nett“ ist (Cohen). In diesem Schreibprojekt sei an dieser Stelle der Beitrag von Jan Hodel emp- 
fohlen; allgemeiner außerdem Tim Hitchock „Academic History Writing and its Disconnects" (journalofdigitalhumanities.org/1-1/academic-historywriting-and-its-disconnects-by-tim-hitchcock/ - besonderes Reflexionen zum Schreibprozess unter verschiedenen Review-Bedingungen, in: „A postendum").

Trefflich ist in diesem Zusammenhang auch der Hinweis von Dan Cohen auf die "The tyranny of the calendar": "There's too much attention to chronology rather than content and the associations between that content." Denn das mag die Kehrseite der zeitlichen Dimension sein, die ins Schreibsystem Weblog Einzug gehalten hat: Rasch schielen wir auf die Quantität der Einträge, anstatt die Qualität von wenigen Publikationen nur alle paar lange Wochen zu würdigen. Aber die Ökonomie der Aufmerksamkeit lässt sich nicht nur lenken von Qualität.

\section{Die Macht der Portale}

Bloggen sollte eigentlich sein: freies Publizieren, frei zugänglich, gewichtet durch die veröffentlichten Inhalte und die - nach und nach - generierten Verlinkungen.

Unterstützung anzunehmen und anzufordern im Bemühen, Aufmerksamkeit zu generieren, ist nicht nur legitim, sondern klug. Als institutionelle Distributoren sind vorhandene Online-Plattformen des Fachs ebenso denkbar wie Verknüpfungen mit kommerziellen Angeboten - wie sie etwa Verlage zur Verfügung stellen. Beide Varianten haben Vor- und Nachteile. Beide sind nicht per se „neutral“ oder "unabhängig“ - letzteres, im Sinne von institutioneller und finanzieller Unabhängigkeit können bedingt digitale Monolithe wie hist.net, historyonics o.ä. für sich proklamieren, ersteres niemand. Wissenschaftliche Verlage haben die Blogosphäre sehr lange ignoriert, sie verlinken nun auf Blogs „ihrer" AutorInnen und entwickeln gemeinsame, thematisch abgegrenzte Projekte. Forschungsinstitute veröffentlichen Gemeinschaftsblogs oder verlinken wiederum.

Einzigartig ist derzeit die Aufmerksamkeitsmaschine, die de.hypotheses.org sehr erfolgreich anwirft (siehe Beitrag von Mareike König). Anknüpfend an das Mutterschiff aus Frankreich und vernetzt mit mehr und mehr europaweiten Beibooten zielt de.hypotheses.org darauf, die zentrale Plattform auch für deutschsprachige geschichtswissenschaftliche Blogs zu werden. Hinter dem gesamten hypotheses-Projekt steht eine bemerkenswerte (Man)Power und es ist unbedingt erfreulich, dass die Text-Form geisteswissenschaftliches Blog Schwung und Dynamik entwickelt, die vorher lange vermisst wurden. hypotheses.org arbeitet 
jedoch nicht mit Verlinkungen auf Blogs, die außerhalb von hypotheses angesiedelt sind. Wer mitmachen will, muss auf hypotheses umziehen, und das heißt vor allem auch: eine hypotheses-URL annehmen. Hypotheses bietet neben Serverkapazitäten und geordneter Präsentation auch technischen Support: Wer den eigenen Blog bei hypotheses aufsetzen will, wird dankbar das vorstrukturierte und angepasste WordPress in Anspruch nehmen - muss es aber auch, denn hypotheses arbeitet ausschließlich mit dieser vereinheitlichten und vereinheitlichenden Software (zum für und wider: Beitrag Michael Schmalenstroer).

Das demokratische, kollektive Prinzip des Bloggens wird von de.hypotheses bisher noch weitgehend ausgeklammert. Es werden zwar Veränderungen angekündigt, zum 1. Geburtstag gab es auch eine Möglichkeit die fünf besten Blogs oder Beiträge zu küren. Aber bisher unterliegt nicht nur die Auswahl, wer mitmachen darf, sondern auch welcher Blog auf die Startseite von hypotheses.org gestellt wird, der Redaktion, nicht etwa der Community. Es ist richtig, dass die Auswahl der Aufsätze in geschichtswissenschaftlichen Zeitschriften, beispielweise der „Historischen Zeitschrift", auch von einer Peer Group, ggf. im Peer Review getroffen wird. Die Kommunikationsart „Bloggen“ tritt jedoch meist gerade als Gegenmodell zu solchen traditionellen Publikationsweisen an. Doch wie bei Fachzeitschriften wurde auch die Redaktion von hypotheses nicht gewählt und kann auch nicht von der Community nach- oder umbesetzt werden. Bezeichnender Weise ist die Seite von hypotheses selbst, die ja wie eine BlogKollektion funktioniert, nicht kommentierbar. Als Kommunikations-Medium gibt es nur die E-Mail in die Black-Box der Redaktion; und den RedaktionsBlog. Dabei sollte auch die Kommunikation zu den einzelnen Blogs zusammengefasst werden, zu einem echten Netzwerk. Das hieße z. B., dass man die Kommentare der annotierten Blogs auf der Startseite mit laufen lässt.

Dass auch andere Wege beschritten werden können, zeigen der Idee nach die ersten Ansätze von Global Perspectives on Digital History. Auch hier ist ein starkes, bestimmendes Redaktions- und Herausgeberteam maßgeblich. Auch die Editoren von GPDH wählen einen „Blogs des Monats“ oder Ähnliches aus; parallel soll jedoch die von RSS-Feeds gespeiste Präsentation der historyblogosphere auf gpdh.org/global-perspectives-unfiltered/ laufen - derzeit in reduzierter Form auf gpdh.org/. In der Umsetzung gibt es leider noch viele Lücken und es könnte auch noch weiter gedachtet werden in Richtung Unterstützung der Auswahl durch semantische Algorithmen, wie es schon längere Zeit The Early Modern Commons praktiziert (und dabei sogar ohne WordPress arbeitet): commons.earlymodernweb.org/. Maßgeblicher Unterschied zu hypotheses ist jedoch, dass auf die vernetzten Blogs verlinkt wird, sie müssen nicht ins GPDH-Boot einsteigen, sondern dürfen selbständig weitersegeln - gerne auch unter hypotheses. Dafür ist andererseits aber auch keine dauerhafte Verbindung gegeben. Während die AutorInnen auf hypotheses einen dauerhaften Platz auf 
der Plattform haben, müssen sie sich diesen bei GPDH mit jedem Blog-Eintrag neu erschreiben.

Interessant ist die Frage, warum es noch keine vollständig von der Netzgemeinde gesteuerte Plattform gibt. Ansätze finden sich zwar beim Journal of Digital Humanities: journalofdigitalhumanities.org/. Eines der Kriterien, nach denen die Beiträge zur Publikation (hier allerdings im Rahmen einer Open Access Zeitschrift) auswählt werden, sind Response und Feedback, die sie von der digitalen Community erhalten, und so ist das Journal mit einem starken Manifest angetreten:

„We wish to underline this notion of community. Indeed, this new journal is predicated on the idea that high-quality, peer-reviewed academic work can be sourced from, and vetted by, a mostly decentralized community of scholars rather than a centralized group of publishers."

Allerdings ist auch bei JDH der Wahl durch die „community“ noch ein „editor's choice“"vorgeschaltet, die eine Art Best Of im Format Digital Humanities Now lanciert.

Die ausschließliche Steuerung durch die Netzgemeinde scheint also noch immer nicht zu funktionieren. Die Sorge vor, oder auch die reale Gefahr von zu viel Spam, zu vielen unsachlichen Beiträgen, zu viel ich-gesteuerter Promotion von eigentlich belanglosen Inhalten, machen die derzeit gängigen Arten von Plattformen attraktiv.

\section{Und schließlich ...}

Die letzten Überlegungen zielten eher in Richtung Publizieren als Schreiben von Geschichte, denn in einem kollektiven, digitalen Schreibprozess sind beide Ebenen nicht länger voneinander zu trennen, sondern müssen gemeinsam betrachtet werden.

Wenn das Ich und das Wir im Schreibprozess mehr Gewicht bekommen, so wächst die Hoffnung, dass Ausmaß, aber durchaus auch Muße zur Selbstreflexion des eigenen Schreibens zunehmen. Diese Selbstreflexion muss natürlich die Eigenheiten, auch die Auswahl des Mediums einbeziehen, und darf sich bei aller Euphorie keine Narrenkappe überziehen. Experimentierfreude ist wunderbar und hat dem Feld der (deutschsprachigen) Geschichtsschreibung bisher sicherlich eher gefehlt. Wenn sie als ein weiteres Charakteristikum der Blogosphäre durch diese Tür auch in die Geschichtsschreibung und -wissenschaft ein bisschen einzieht, ist das unbedingt begrüßenswert. Zu aller Freude am Neuen und am Ausprobieren muss sich zugleich die stete Reflexion über das eigene Forschen, Schreiben, Publizieren gesellen. 


\section{Die zitierten Blog-Beiträge bzw. Web-Seiten}

Dan Cohen, Creating a Blog from Scratch. Part 2: Advantages and Disadvantages of Popular Blog Software, Blogbeitrag, 18.12.2005, in: Blog: Dan Cohens Digital Humanities Blog [www.dancohen.org/2005/12/18/creating-ablog-from-scratch-part-2-advantages-and-disadvantages-of-popular-blogsoftware/], eingesehen, 4.9.2012.

Tim Hitchock historyonics, historyonics.blogspot.de/ 27.1.2013.

Newton Key, The Early Modern England, earlymodernengland.blogspot.co. uk/.Lilian Landes, Hierarchie meets Netz meets Wissenschaft, Blogbeitrag, 17.8.2012, in: Blog: Rezensieren, Kommentieren, Bloggen [rkb.hypotheses.org/ 194], eingesehen 4.9.2012.

Andreas Lerch, Wozu ein Blog?, Blogbeitrag, 10.6.2012, in: Blog: Astrologie in der Frühen Neuzeit [astrologiefnz.hypotheses.org/35], eingesehen 2.10.2012.

Marc Mudrak, Dissertationsprojekt, Unterseite, in: Altgläubige in der Reformation [catholiccultures.hypotheses.org/dissertationsprojekt], eingesehen 2.10.2012.

The Early Modern Commons, Blog [commons.earlymodernweb.org/], eingesehen 29.9.2012.

Open Review. A Study of Contexts and Practicis, ed. Kathleen Fitzpatrick u. a. [mediacommons.futureofthebook.org/mcpress/open-review/], eingesehen: 27.1.2013.

Thomas Steinfeld, Why Digital Humanities is "Nice", 25.5.2010, in Blog: Found History [www.foundhistory.org/2010/05/26/why-digital-humanitiesis-\%E2\%80\%9Cnice\%E2\%80\%9D/], eingesehen 15.10.2012.

Jan Hecker-Stampehl, Warum dieser Blog, Blogbeitrag 11.2011, in: Blog: Nordic History [nordichistoryblog.hypotheses.org/59], eingesehen 20.9.2012.

Robert Zimmermann, Über das umstrittene Gedächtnis, Unterseite, in: Blog: Das umstrittene Gedächtnis. Erinnerungspraktiken in Skandinavien, [umstrittenesgedaechtnis.hypotheses.org/uber], eingesehen 2.10.2012.

Writing History in the Digital Age, ed. edited by Jack Dougherty and Kristen Nawrotzki [writinghistory.trincoll.edu/], eingesehen: 27.1.2013. 


\section{Zeitschriftenartikel}

A Community-Sourced Journal (editors), in: Journal of Digital Humanities, Vol. 1 No. 1 Winter 2011 [journalofdigitalhumanities.org/1-1/a-communitysourced-journal/], eingesehen 12.10.2012.

Tim Hitchock „Academic History Writing and its Disconnects“, in: Journal of Digital Humanites, Vol. 1 No. 1 Winter 2011 [journalofdigitalhumanities.org/11/academic-history-writing-and-its-disconnects-by-tim-hitchcock/], eingesehen 3.3.2013. 
